\title{
Bacteriological Spectrum of Neonatal Septicemia in Neonatal Intensive Care Unit
}

\author{
K. Sajitha* \\ Department of Microbiology, Dr B R Ambedkar Medical College, Bengaluru, India \\ *Corresponding author
}

\begin{abstract}
A B S T R A C T

\begin{tabular}{|l|}
\hline Keyw or d s \\
Bacteriological \\
spectrum, Neonatal \\
septicaemia, \\
Neonatal intensive \\
care unit \\
\hline Article Info \\
\hline Accepted: \\
04 April 2019 \\
Available Online: \\
10 May 2019 \\
\hline \hline
\end{tabular}

Neonatal septicemia either acquired from the mother (vertical transmission) or nosocomial (horizontal transmission) is a forbidable problem encountered in the neonatal unit and is the commonest primary cause of morbidity and mortality among neonates. The present study was conducted in Department of Microbiology, Dr B R Ambedkar Medical College, Bengaluru, over a period of one year from June 2016 - May 2017. Blood sample were collected from 100 clinically suspected cases of neonatal septicemia admitted in the neonatal intensive care unit. Of the 100 cases studied, 59 were males and 31 were females. Among 34 culture positive neonates 22 were male and 12 were females. Majority i.e. $34 \%$ of the isolates were Gram negative organisms, Klebsiella pneumoniae being the commonest isolated in 11 of the 34 culture positive cases followed by Pseudomonas aeruginosa 07 . The other organisms isolated were Escherichia coli 01, Proteus vulgaris 01, Citrobacter freundii 01. Gram positive organisms were obtained in 9\% out of 34 cases. Coagulase negative Staphylococci 03 was commonest isolate followed by Staphylococcus aureus 01 cases. This study concludes that empiric therapy for suspected neonatal septicemia should cover both Gram-negative bacilli and Gram-positive cocci particularly Klebsiella pneumoniae and Staphylococcus aureus.
\end{abstract}

\section{Introduction}

Neonatal septicemia either acquired from the mother (vertical transmission) or nosocomial (horizontal transmission) is a forbidable problem encountered in the neonatal unit and is the commonest primary cause of morbidity and mortality among neonates. The early and efficient clinical diagnosis of neonatal bacterial sepsis remains a difficult task as the clinical signs and symptoms of septicemia are vague and nonspecific, hence one depends upon the laboratory diagnosis yet, to treat neonates with antibiotics presumptively on the basis of subtle signs and risk factors alone are likely to result in over treatment. ${ }^{1}$

Traditional methods such as blood culture requires a minimum of 48 hours for the diagnosis to be made, certain rapid diagnostic tests were developed to diagnose bacteremia so that suitable antibiotic treatment can be started at early to prevent morbidity and mortality. $^{2}$

The overall incidence of neonatal septicemia remained fairly constant over the past 4-5 decades with little change. The incidence 
varies from one institution to another and in same institution it varies from time to time which depends on various predisposing factors like low birth weight, prematurity, obstetric and nursing practices. A higher incidence is observed by many authors in developing countries than the developed nations. The reasons listed are high rate of home delivery, often overseen by unskilled attendants, unclean environment and limited resources. $3,4,5$

The incidence has been reported to vary between 1-10/1000 live births in developing countries and 1-4/1000 live births in developed countries. ${ }^{6}$ Neonatal septicemia continues to be major cause of neonatal morbidity and mortality. Although the incidence of neonatal septicemia has shown a steady decline in west, it continues to be among the four leading causes of neonatal mortality in India accounting for one fourth to nearly half of all neonatal deaths.

The main objectives of this study the bacteriological spectrum of Neonatal Septicemia in Neonatal Intensive Care Unit

\section{Materials and Methods}

The present study was conducted in Department of Microbiology, DR B R Ambedkar Medical College, Bengaluru, over a period of one year from June 2016 - May 2017. Blood sample were collected from 100 clinically suspected cases of neonatal septicemia admitted in the neonatal intensive care unit.

\section{Inclusion criteria}

All clinically suspected septicemia neonates on the basis of

Maternal fever

Foul smelling lochia

Prolong labour
Suspected neonates with signs and symptoms of sepsis

\section{Exclusion criteria}

Neonates with gross congenital anomalies and clinical feature suggestive of septicemia receiving antibiotics were excluded from the study.

\section{Results and Discussion}

Of the 100 cases studied, 59 were males and 31 were females.

Among 34 culture positive neonates 22 were male and 12 were females

Majority i.e. 34\% of the isolates were Gram negative organisms, Klebsiella pneumoniae being the commonest isolated in 11 of the 34 culture positive cases followed by Pseudomonas aeruginosa 07.

The other organisms isolated were Escherichia coli 01, Proteus vulgaris 01, Citrobacter freundii 01.

Gram positive organisms were obtained in $9 \%$ out of 34 cases. Coagulase negative staphylococci 03 was commonest isolate followed by Staphylococcus aureus 01 cases.

Of the 100 cases studied, 59 were males and 31 were females. Among 34 culture positive neonates 22 were male and 12 were females. Majority i.e. $34 \%$ of the isolates were Gram negative organisms, Klebsiella pneumoniae being the commonest isolated in 11 of the 34 culture positive cases followed by Pseudomonas aeruginosa 07. The other organisms isolated were Escherichia coli 01, Proteus vulgaris 01, Citrobacter freundii 01. Gram positive organisms were obtained in $9 \%$ out of 34 cases. Coagulase negative staphylococci 03 was commonest isolate followed by Staphylococcus aureus 01 cases.

These results are comparable with the observations made by Tallur et al., (63.60\%), Uddin Ahmed et al., (63.00\%), Movahedin et 
al., (71.2\%), Shrestha et al., (75\%), Shansanam Gheibi et al., (55.03\%), Haider shirazi et al., (59\%) and Ramesh Bhat et al., $(53.7 \%)$.

The study conducted by Anita Sharma et al., (20\%), Kenneth C. Iregbu et al., (22\%) and Joshi et al., (25.80\%) showed a very low positivity rate, While study conducted by Roy et al., (47.50\%), Jaswal et al., (42\%), Betty Chacko et al., (43.10\%), Tallur et al., (64.87\%) and Karthikeyan et al., (51.34\%) showed high culture positivity rate (Table 1 and 2).

Table.1 Age and sex wise distribution of cases

\begin{tabular}{|l|l|l|l|l|l|l|}
\hline \multirow{2}{*}{ Gender } & \multicolumn{2}{|c|}{$\begin{array}{l}\text { Clinically } \\
\text { Suspected }\end{array}$} & \multicolumn{2}{c|}{$\begin{array}{c}\text { Culture } \\
\text { Positive }\end{array}$} & \multicolumn{2}{c|}{$\begin{array}{c}\text { Culture } \\
\text { Negative }\end{array}$} \\
\cline { 2 - 8 } & No & $\mathbf{\%}$ & No & $\mathbf{\%}$ & No & $\%$ \\
\hline Males & 59 & 59 & 22 & $\mathbf{2 2}$ & 37 & $\mathbf{3 7}$ \\
\hline Females & 31 & 31 & 12 & 12 & 19 & 19 \\
\hline Total & $\mathbf{1 0 0}$ & $\mathbf{1 0 0}$ & $\mathbf{3 4}$ & $\mathbf{3 4}$ & $\mathbf{5 6}$ & $\mathbf{5 6}$ \\
\hline
\end{tabular}

Table.2 Spectrum of bacterial isolates

\begin{tabular}{|l|c|c|}
\hline \multirow{2}{*}{ Bacterial Isolates } & \multicolumn{2}{c|}{ Culture Positive } \\
\cline { 2 - 3 } & Number & Percentage \\
\hline A) GRAM POSITIVE ISOLATES & & \\
\hline Staphylococcus aureus & 01 & $\mathbf{0 1}$ \\
\hline Coagulase negative staphylococci (CONS) & $\mathbf{0 3}$ & $\mathbf{0 3}$ \\
\hline TOTAL & $\mathbf{0 9}$ & $\mathbf{0 9}$ \\
\hline B) GRAM NEGATIVE ISOLATES & $\mathbf{1 1}$ & $\mathbf{1 1}$ \\
\hline Klebsiella pneumoniae & 07 & $\mathbf{0 7}$ \\
\hline Pseudomonas aeruginosa & 01 & $\mathbf{0 1}$ \\
\hline Escherichia coli & 01 & $\mathbf{0 1}$ \\
\hline Proteus vulgaris & 01 & $\mathbf{0 1}$ \\
\hline Citrobacter freundii & $\mathbf{3 4}$ & $\mathbf{3 4}$ \\
\hline TOTAL & & \\
\hline
\end{tabular}

Stoll B J reported a predominance of Gram negative septicemia (21\%-85\%) over Gram positive septicemia. ${ }^{7}$ Tallur et al., (2000) reported an higher incidence of 37.6/1000 live births in their study among hospital born babies and that the incidence was higher among pre mature and low birth weight babies. ${ }^{8}$

Agnihotri et al., (2004) reported that the incidence of Gram negative and Gram positive organisms was $58.5 \%$ and $41.5 \%$ respectively. ${ }^{9}$ Madhu Sharma et al., in their study conducted at Rohtak observed frequency of $22.9 \%$ neonatal sepsis among 4368 neonates. ${ }^{10}$

Rajlakshmi Viswanathan et al., (2011) reported that the incidence of culture proven neonatal sepsis among inborn babies was 14.8/1000 live births. The proportion of culture positive sepsis for out born babies admitted in NICU was $8.3 \% .^{11}$

Observation from the past 30 year literature have shown that males had approximately 2-6 
fold higher incidence of septicemia compared to females accounting for $59-82 \%$ of cases. This may be related to $\mathrm{X}$ linked immune regulatory genes that regulate the synthesis of Y-globulins. As males have only one $\mathrm{X}$ chromosome, they are immunologically more susceptible to infection than the female. Infections in males were found to be significantly culture positive with predominance of Gram negative bacterial septicemia. $^{12}$

Tallur et al., ${ }^{8}$ noted a high incidence of Staphylococcus spp in their study, pointing towards its nosocomial origin. All these isolates were $100 \%$ susceptible to Vancomycin. In the study by Karthikeyan major isolate was Staphylococcus aureus and most of these isolates belonged to early onset septicemia group; 24/59(40.7\%).

This study concludes that empiric therapy for suspected neonatal septicemia should cover both Gram-negative bacilli and Gram-positive cocci particularly Klebsiella pneumoniae and Staphylococcus aureus. An effective infectioncontrol programme, regular antibiotic susceptibility surveillance and evaluation, and the enforcement and periodic review of the antibiotic policy of the hospital as well as the encouragement of rational antibiotic use will reduce the rates of acquiring nosocomial infections and development of bacterial resistance.

\section{References}

1. Jeffery, S., Gerdes, Richard Polin. "Early Diagnosis and Treatment of Neonatal Sepsis". Indian J Pediatric 1998; 65(1):6378.

2. Madhubala Parikh, Nandan Singh. "Rapid diagnosis of neonatal bacteraemia". Indian Journal of Medical Microbiology 1995; 13:

\section{$37-40$}

3. Neelam Kaistha, Manjula Mehta, Nidhi Singla, Ritu Garg, Jagdish Chander. "Neonatal septicemia isolates and resistance patterns in a tertiary care hospital of North India". J Infect Dev Ctries 2010; 4(1):055-057.

4. Khatua, SP, Das AK, Chatterjee BD, Khatua S, Ghose B, Saha A. "Neonatal septicemia”. Indian J Pediatr 1986; 53(4): 509-14.

5. Mathur, NB., Khalil A, Sarkar R, Puri RK. "Mortality in neonatal septicemia with involvement of mother in management". Indian Pediatrics 1991; 28: 1259-63.

6. Jane, D., Siegel, George H. McCracken Jr. Sepsis Neonatorum. New Engl J.Med 1981 Mar; (11): 642-7.

7. Stoll BJ. "The global impact of neonatal infection". Clin Perinatol 1997; 24:1- 21.

8. Shashikala, S., Tallur, Kasturi AV, Shobha D. Nadgir, Krishna BVS. "Clinico bacteriological Study of Neonatal Septicemia in Hubli". Indian J Pediatr 2000; 67(3):169-74.

9. Nalini Agnihotri, Neelam Kaistha, Varsha Gupta. "Antimicrobial Susceptibility of Isolates from Neonatal Septicemia". Jpn. J. Infect. Dis 2004; 57:273-75.

10. Madhu Sharma, Nidhi Goel, Uma Chaudhary, Ritu Aggarwal, Arora DR. "Bacteremia in Children". Indian J Pediatr 2002 Dec; 69 (12): 1029-32.

11. Rajlakshmi Viswanathan, et al., "A etiology and Antimicrobial resistance of neonatal Sepsis at a Tertiary Care Centre in Eastern India: A 3 year Study". Indian J Pediatr 2011April; 78(4):409-12.

12. Betty Chacko, Inderpreet Sohi. "Early Onset Neonatal Sepsis". Indian J Pediatr 2005 Jan; 72(1): 23-6.

\section{How to cite this article:}

Sajitha, K. 2019. Bacteriological Spectrum of Neonatal Septicemia in Neonatal Intensive Care Unit. Int.J.Curr.Microbiol.App.Sci. 8(05): 279-282. doi: https://doi.org/10.20546/ijcmas.2019.805.032 